\title{
Lesões orais em pacientes com COVID-19: uma síntese de evidências atuais
}

\author{
Oral injuries in patients with COVID-19: \\ a synthesis of current evidence
}

\author{
José Carlos Costa dos Santos Júnior ${ }^{1}$ (i) \\ Marla Sobral de Souza ${ }^{2}$ (1) \\ Vanderlam Souza dos Santos ${ }^{3}$ (1)
}

\section{João Matheus Silva Carvalho 4 (i) Alessandra Lais Pinho Valente Pires ${ }^{5}$ (1) Cristiane Brandão Santos Almeida ${ }^{6}$ (1)}

\begin{abstract}
${ }^{1}$ Autor para correspondência. Unidade de Ensino Superior da Feira de Santana (Feira de Santana). Bahia, Brasil. jozesant82@gmail.com 2,3Unidade de Ensino Superior da Feira de Santana (Feira de Santana). Bahia, Brasil. msobraldesouza@gmail.com, vanderlamsouza3@gmail.com, ${ }^{4}$ Unidade de Ensino Superior da Feira de Santana (Feira de Santana). Bahia, Brasil. mateuscarvalho520@gmail.com ${ }^{5}$ Faculdade São Leopoldo Mandic (Campinas), Unidade de Ensino Superior da Feira de Santana (Feira de Santana), Universidade Estadual de Feira de Santana (Feira de Santana). São Paulo, Bahia, Brasil. lecavalent@hotmail.com ๔Unidade de Ensino Superior da Feira de Santana (Feira de Santana), Universidade Estadual de Feira de Santana (Feira de Santana). Bahia, Brasil.
\end{abstract} cristianebsalemida@gmail.com
RESUMO |OBJETIVO: Descrever as principais manifestações orais em pacientes com COVID-19. MÉTODO: Trata-se de uma revisão integrativa realizada nas bases de dados do BVS e do MEDLINE/Pubmed, em que foram incluídos artigos científicos em Espanhol e Inglês até o período de setembro de 2020. RESULTADOS: As principais manifestações relacionadas ao COVID-19 foram: infecções fúngicas, infecção recorrente do vírus herpes simplex (HSV-1), ulcerações orais inespecíficas, erupções fixas de medicamentos, disgeusia, xerostomia ligada à diminuição do fluxo salivar e gengivite - como resultado do comprometimento do sistema imunológico e/ou mucosa oral suscetível. CONCLUSÃO: Ressalta-se a importância de um minucioso exame clínico pelo cirurgião-dentista para diagnóstico e tratamento dessas alterações orais.

PALAVRAS-CHAVE: COVID-19. Manifestações orais. Odontologia. Mucosa bucal.

\begin{abstract}
OBJECTIVE: To describe the main oral manifestations in patients with COVID-19. METHOD: This is an integrative review carried out in the VHL and MEDLINE / Pubmed databases, in which scientific articles were included in Spanish and English until the period of September 2020. RESULTS: The main manifestations related to COVID-19 were: fungal infections, recurrent infection of the herpes simplex virus (HSV-1), nonspecific oral ulcerations, fixed drug eruptions, dysgeusia, dry mouth linked to decreased salivary flow and gingivitis - as a result of impaired immune system and / or susceptible oral mucosa. CONCLUSION: The importance of a thorough clinical examination by the dentist for the diagnosis and treatment of these oral changes is emphasized.
\end{abstract}

KEYWORDS: Coronavirus infections. Oral manifestations. Dentistry. Mouth mucosa. 


\section{Introdução}

A nova doença de coronavírus 2019 (COVID-19) tornou-se uma ameaça importante e urgente à saúde global. No início de 2020, o novo coronavírus, inicialmente nomeado 2019-nCoV, foi renomeado pela Organização Mundial da Saúde (OMS) para Síndrome Respiratória Aguda Grave Coronavírus-2 (SARSCoV-2 ${ }^{1,2}$. A facilidade de transmissão deste vírus resultou em uma pandemia, com aceleração do número de casos e milhares de óbitos em praticamente todo o mundo ${ }^{3}$. A OMS confirmou 16.114.449 casos e 646.641 mil óbitos até o dia 27 de julho de 20201.

A COVID-19 é transmitida de pessoa para pessoa, por gotículas de saliva, espirro, tosse, assim como pelo contato da boca, nariz ou olhos, ou até mesmo, por meio de objetos e superfícies contaminadas. Embora um quadro clínico grave seja mais provável em indivíduos com comorbidades, pessoas de todas as idades são susceptíveis à doençâ4.

Além dos sintomas mais comuns, como tosse seca, pirexia, dispneia, fadiga e algia muscular, estudos têm evidenciado algumas manifestações orais em pacientes com COVID-195-9. Ainda não se sabe se essas manifestações são resultantes da infecção viral direta ou mesmo da debilidade sistêmica, uma vez que são susceptíveis à infecções oportunistas e também a reações adversas aos tratamentos medicamentosos 2,10 .

Pesquisas atuais expõem que os danos do novo coronavírus às vias respiratórias e a outros órgãos podem estar relacionados à distribuição de receptores da enzima conversora de angiotensina 2 (ECA-2). Dessa forma, células com receptor ECA-2 podem se tornar hospedeiras do vírus e causar reações inflamatórias em órgãos e tecidos relacionados, como a mucosa da língua e glândulas salivares 2,11 .

As investigações acerca das possíveis correlações da ação patogênica do SARS-CoV-2 sobre as células epiteliais e nervosas, com as manifestações extrapulmonares desta infecção na região de cabeça e pescoço, em especial a cavidade oral, são recentes. Portanto, o objetivo deste estudo é fazer uma síntese de evidências científicas sobre as principais manifestações orais em pacientes com COVID-19.

\section{Método}

Este estudo consiste em uma revisão integrativa da literatura, em que foram consultados artigos científicos indexados nos portais eletrônicos da Biblioteca Virtual em Saúde (BVS) e PubMed.

\section{Critérios de elegibilidade}

A coleta dos dados foi realizada entre o mês de juIho e setembro de 2020, conforme os critérios de inclusão: artigos científicos disponíveis na íntegra, sem restrição quanto ao tipo de estudo, nos idioma Espanhol e Inglês e publicados até setembro de 2020. Foram excluídos àqueles que não seguiram os critérios (idioma, período de publicação e texto disponível na íntegra), além dos que não estavam relacionados com o tema e os duplicados. 


\section{Fontes de informações e estratégia de busca}

Para a seleção dos artigos nas bases de dados, inicialmente foram consultados no MeSH (Medical Subject Headings) e no DeCS (Descritores em Ciências da Saúde) os seguintes descritores de busca: "Coronavirus Infections"; "COVID-19"; "Betacoronavirus"; "Dentistry"; "Mouth mucosa"; "ACE-2 enzyme"; "Oral manifestations", e seus correspondentes em espanhol - "Infecciones por Coronavirus"; "COVID-19"; "Betacoronavirus"; "Odontología"; "Mucosa Bucal"; "ACE-2 enzima"; "Manifestaciones Bucales".

Como estratégia de busca, os operadores booleanos AND e OR foram utilizados para compor as combinações entre os descritores (Quadro 1).

Quadro 1. Estratégias de busca

\begin{tabular}{|c|c|c|}
\hline COMBINAÇÃO & ESTRATÉGIA DE BUSCA EM INGLÊS & ESTRATÉGIA DE BUSCA EM ESPANHOL \\
\hline COMBINAÇÃO 1 & $\begin{array}{c}\text { (Coronavirus Infections OR } \\
\text { Betacoronavirus) AND Dentistry AND } \\
\text { Oral manifestations }\end{array}$ & $\begin{array}{c}\text { (Infecciones por Coronavirus OR } \\
\text { Betacoronavirus) AND Odontología } \\
\text { AND Manifestaciones Bucales }\end{array}$ \\
\hline COMBINAÇÃO 2 & COVID-19 AND Oral manifestations & COVID-19 AND Manifestaciones Bucales \\
\hline COMBINAÇÃO 3 & $\begin{array}{c}\text { Coronavirus Infections AND (Oral } \\
\text { manifestations OR Mouth mucosa) }\end{array}$ & $\begin{array}{c}\text { Infecciones por Coronavirus AND } \\
\text { (Manifestaciones Bucales OR Mucosa } \\
\text { Bucal) }\end{array}$ \\
\hline COMBINAÇÃO 4 & COVID-19 AND Mouth mucosa AND ACE- & $\begin{array}{c}\text { COVID-19 AND Mucosa Bucal AND ACE- } \\
\text { 2 enzimame }\end{array}$ \\
\hline
\end{tabular}

\section{Seleção dos estudos}

Os revisores realizaram a triagem dos artigos por meio da leitura do título e resumo, de maneira independente. Todos os artigos que foram triados tiveram sua elegibilidade confirmada pela leitura do texto na íntegra. Além disso, foi feita busca manual e cruzamento de referências dos artigos elegíveis.

\section{Resultados}

Foram identificados 96 artigos a partir das bases de dados selecionadas (63 no PubMed e 33 na BVS). Depois que os artigos em duplicatas foram excluídos e, mediante a leitura dos títulos e resumos, 28 artigos foram selecionados para leitura completa. Apenas 20 artigos obedeceram aos critérios de elegibilidade da revisão integrativa (Figura 1). 
Figura 1. Fluxograma da pesquisa com critérios de seleção adaptado do PRISMA (Preferred Reporting Items for Systematic Reviews and Meta-Analyses)

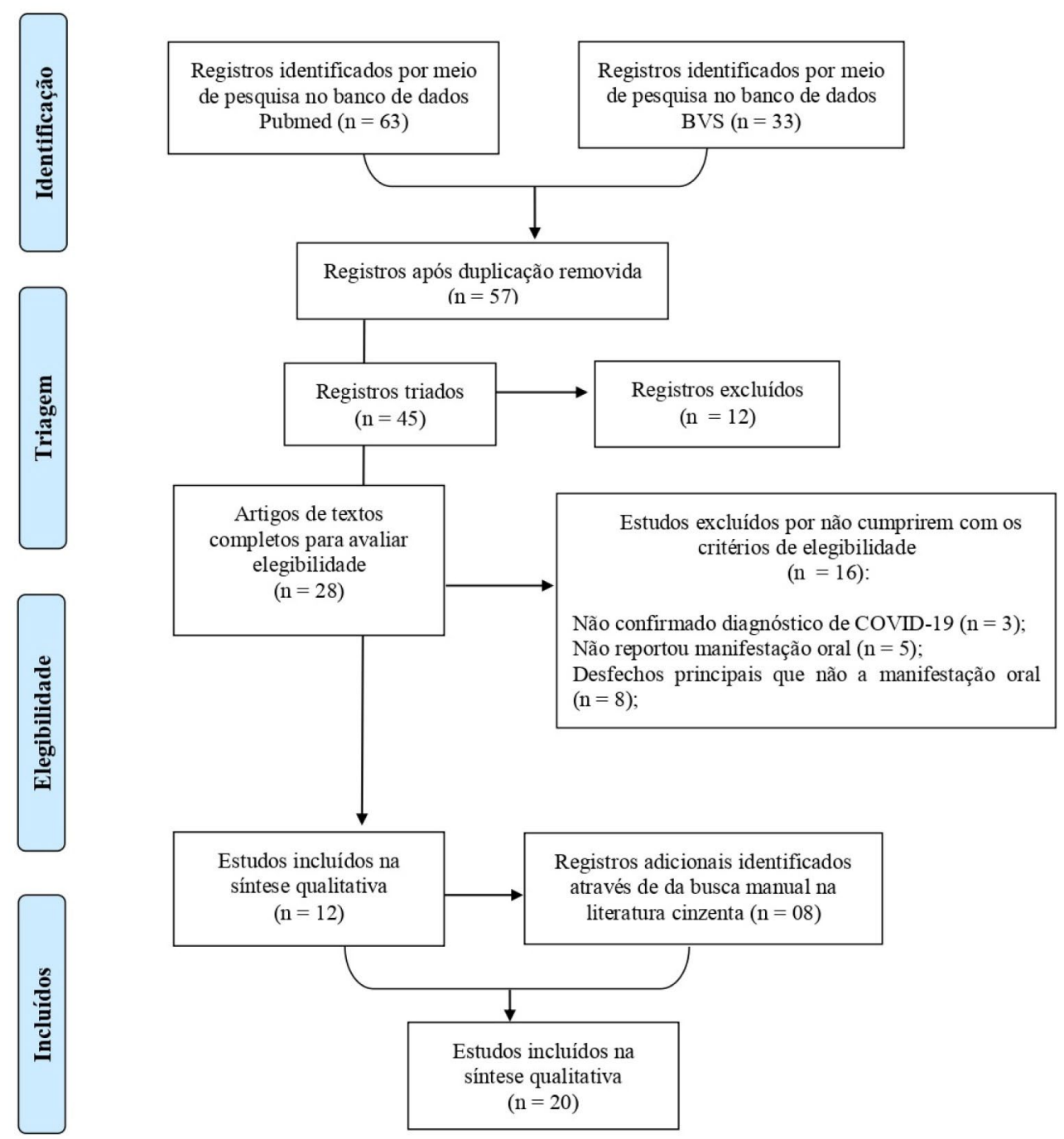

As principais características dos 20 estudos elegíveis estão organizadas no Quadro 2. Pode-se observar que a maioria dos estudos era de relato de caso clínico (08) e carta ao editor (03); publicados no mês de maio e junho (ambos com 07), no idioma inglês (19) e na região de Oriente Médio (07) e Ásia (05). 


\begin{tabular}{|c|c|c|c|c|}
\hline AUTORES & IDIOMA & $\begin{array}{c}\text { MÊS DE } \\
\text { PUBLICAÇÃO }\end{array}$ & $\begin{array}{l}\text { DESENHO DO } \\
\text { ESTUDO }\end{array}$ & $\begin{array}{l}\text { REGIÃO DO } \\
\text { ESTUDO }\end{array}$ \\
\hline Abanoub, Marwa, $2020^{18}$ & Inglês & Maio & Carta ao editor & Oriente Médio \\
\hline Abu-Hammad, $2020^{21}$ & Inglês & Maio & Carta ao editor & Oriente Médio \\
\hline Al-khatib, $2020^{19}$ & Inglês & Maio & Carta ao editor & Oriente Médio \\
\hline Ansari et al., $2020^{25}$ & Inglês & Junho & Relato de Caso & Oriente Médio \\
\hline Biadsee et al., $2020^{7}$ & Inglês & Maio & Relato de Caso & Oriente Médio \\
\hline Chauhan et al., $2020^{6}$ & Inglês & Junho & Hipótese & Ásia \\
\hline Chaux-Bodard et al., $2020^{5}$ & Inglês & Abril & Relato de Caso & Europa \\
\hline Herrera et al., $2020^{10}$ & Inglês & Junho & Revisão bibliográfica & Europa \\
\hline Odeh et al., $2020^{17}$ & Inglês & Junho & Revisão bibliográfica & Oriente Médio \\
\hline Patel et al., $2020^{27}$ & Inglês & Maio & Relato de Caso & Oriente Médio \\
\hline Rodriguez et al., $2020^{8}$ & Inglês & Setembro & Relato de Caso & Europa \\
\hline Santos et al., $2020^{2}$ & Inglês & Junho & Relato de Caso & América Latina \\
\hline Santos et al.,202020 & Inglês & Setembro & Revisão sistemática & América Latina \\
\hline Sakaida et al., $2020^{26}$ & Inglês & Junho & Relato de Caso & Ásia \\
\hline Sinadinos, Shelswell, $2020^{12}$ & Inglês & Junho & Relato de Caso & Europa \\
\hline Song et al., $2020^{11}$ & Inglês & Maio & Revisão sistemática & Ásia \\
\hline Varadarajan et al., $2020^{22}$ & Inglês & Julho & Hipótese & Ásia \\
\hline Vieira, $2020^{9}$ & Inglês & Maio & Estudo descritivo & $\begin{array}{l}\text { América do } \\
\text { Norte }\end{array}$ \\
\hline Tuñon e Castro-Ruiz, $2020^{18}$ & Espanhol & Abril & Protocolo & América Latina \\
\hline Wang et al., $2020^{24}$ & Inglês & Abril & Estudo retrospectivo & Ásia \\
\hline
\end{tabular}

Nos estudos de relato de caso, os indivíduos apresentam média de idade de 55 anos, acometendo ambos os sexos. Quanto às manifestações orais, a disgeusia, xerostomia e candidíase foram as mais comuns (Tabela 1).

As manifestações orais no estudo de Santos et al. (2020)2 foram observadas durante o internamento hospitalar do paciente, assim como em um dos casos relatados por Rodriguez et al. (2020)ํ. No entanto, neste mesmo estudo houve um relato de aparecimento das lesões após alta hospitalar. Os casos descritos por Sinadinos e Shelswell $(2020)^{12}$, bem como Biadsee et al. (2020)ㄱ não estavam em internamento. 
Tabela 1. Principais manifestações orais em indivíduos portadores da COVID-19 nos estudos de relato de casos

\begin{tabular}{|c|c|c|c|c|}
\hline AUTORES & IDADE & SEXO & LOCAL ANATÔMICO & MANIFESTAÇÕES ORAIS \\
\hline $\begin{array}{l}\text { Ansari et al., } \\
2020^{25}\end{array}$ & $56 ; 75$ & $\begin{array}{l}\text { Masculino; } \\
\text { Feminino }\end{array}$ & Língua e palato duro & $\begin{array}{l}\text { Pequenas úlceras dolorosas com } \\
\text { margens irregulares na llíngua e } \\
\text { palato duro }\end{array}$ \\
\hline $\begin{array}{l}\text { Biadsee et al., } \\
2020^{7}\end{array}$ & 36 & Feminino & Mucosa Bucal e Face & $\begin{array}{l}\text { Disgeusia, xerostomia, dor } \\
\text { muscular mastigatória e dor facial }\end{array}$ \\
\hline $\begin{array}{c}\text { Chaux-Bodard et } \\
\text { al., } 2020^{5}\end{array}$ & 45 & Feminino & Língua & $\begin{array}{l}\text { Úlcera assintomática irregular no } \\
\text { lado dorsal da língua }\end{array}$ \\
\hline $\begin{array}{l}\text { Santos et al., } \\
2020^{2}\end{array}$ & 66 & Masculino & $\begin{array}{l}\text { Língua, Lábio } \\
\text { inferior, Palato e } \\
\text { Mucosa Jugal }\end{array}$ & $\begin{array}{l}\text { Herpes simples recorrente e } \\
\text { candidíase }\end{array}$ \\
\hline $\begin{array}{l}\text { Sakaida et al., } \\
2020^{26}\end{array}$ & 52 & Feminino & $\begin{array}{l}\text { Lábios e mucosa } \\
\text { bucal }\end{array}$ & $\begin{array}{l}\text { Lesões eritematosas e erosões nos } \\
\text { lábios e mucosa bucal }\end{array}$ \\
\hline $\begin{array}{c}\text { Sinadinos e } \\
\text { Shelswell, } 2020^{12}\end{array}$ & 56 & Masculino & Palato e Faringe & $\begin{array}{l}\text { Lesões de herpes simples e } \\
\text { eritema multiforme }\end{array}$ \\
\hline $\begin{array}{l}\text { Patel et al., } \\
2020^{27}\end{array}$ & 35 & Feminino & $\begin{array}{l}\text { Mucosa bucal e } \\
\text { gengiva }\end{array}$ & $\begin{array}{l}\text { Halitose grave com gengivas } \\
\text { eritematosas e edemasiadas, } \\
\text { papilas necrótcas } \\
\begin{array}{l}\text { sangramento com } \\
\text { provocado }\end{array}\end{array}$ \\
\hline $\begin{array}{c}\text { Rodriguez et al., } \\
2020^{8}\end{array}$ & $43 ; 53 ; 78$ & $\begin{array}{l}\text { Feminino } \\
\text { Masculino } \\
\text { Feminino }\end{array}$ & $\begin{array}{l}\text { Língua, Comissura } \\
\text { Labial, Mucosa Bucal }\end{array}$ & $\begin{array}{l}\text { Apresentou-se } 03 \text { casos: caso } 01 \\
\text { com úlceras, sensação de } \\
\text { queimação e despapilação da } \\
\text { língua; caso } 02 \text { com sensação de } \\
\text { queimação, queilite comissural, } \\
\begin{array}{l}\text { anosmia e disgeusia; caso } 03 \text { com } \\
\text { xerostomia, candidíase } \\
\text { pseudomembranosa e queilite } \\
\text { angular }\end{array}\end{array}$ \\
\hline
\end{tabular}

\section{Discussão}

A população em geral é suscetível em se contaminar pelo SARS-CoV-2, não havendo predileção por sexo, nem faixa etária ${ }^{13}$. Analisando sob a ótica dos índices de transmissão e agravamento da infecção pelo SARS-CoV-2, estudos epidemiológicos recentes revelaram que as pessoas portadoras de hipertensão, diabetes, doença pulmonar obstrutiva crônica e doenças renais têm maior probabilidade de desenvolver as formas mais graves da COVID-19, resultando em maiores taxas de morbimortalidade nessa população .

De acordo com a literatura, a COVID-19 é a uma enfermidade que pode apresentar uma variedade de manifestações clínicas ${ }^{14,15}$, desde respiratórias, com prevalência de 5.1 a $79,6 \% \frac{15}{15}$, orais -1.7 a $8.5 \% \frac{15}{}$, dermatológicas -1.1 a

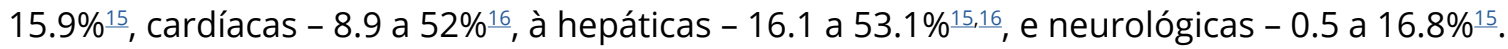

Como a cavidade oral dos pacientes com COVID-19 pode ser afetada, ainda há dúvidas se as manifestações poderiam ser um padrão típico resultante da infecção viral direta. Talvez lesões orais possam ser resultantes de debilidade sistêmica, dada à possibilidade de comprometimento do sistema imunológico e de reações adversas frente ao tratamento médico instituído ${ }^{17}$. Assim, os pacientes podem apresentar infecções fúngicas, infecção recorrente pelo vírus herpes simplex (HSV-1), ulcerações orais inespecíficas, lesões vesiculobolhosas, erupções induzidas por medicamentos, xerostomia ligada à diminuição do fluxo salivar e gengivite descamativa2,17,18. 
A hiposmia/anosmia e a disgeusia/ageusia têm sido reconhecidas recentemente como um dos sintomas mais comuns do COVID-1918. Essa alteração pode ser explicada pelo fato de o SARS-CoV-2 ser conhecido por sua interação com o receptor ECA-2, pois o mesmo facilita a penetração na célula alvo, e esse receptor é amplamente expresso nas células epiteliais da mucosa oral e no cérebro ${ }^{16,19}$. De fato, verificou-se que a expressão de ECA-2 é maior na língua, onde as papilas gustativas são mais abundantes, do que na gengiva ou mucosa bucal. Outra possibilidade é que o SARS-CoV-2 também possa ser detectado em saliva, e a infecção das glândulas salivares também é possível de ocorrer, o que aumenta a disponibilidade do vírus em cavidade oral e sua captação pelas células epiteliais $6,20,21$.

Com este estudo, evidenciou-se que as lesões orais foram descritas em poucos relatos de casos, apresentando controversas quanto ao fato de serem causadas diretamente pelo SARS-CoV-2 ou se representam manifestações secundárias. As manifestações apresentavam aspectos clínicos diversos, como úlceras, bolhas e máculas, variando em quantidade, coloração e localização 2,5,7,8,12. Estudos registraram coinfecções bacterianas e fúngicas em muitos casos fatais de COVID-194,19,20. Os pacientes internados frequentemente recebem antibióticos, no entanto, nenhuma especificação sobre a sensibilidade bacteriana é fornecida ${ }^{15}$. No estudo de Santos et al., (2020) ${ }^{20}$ foram observados lesões na mucosa oral durante a internação de 15 dias, corroborando a hipótese de coinfecções, comprometimento da imunidade ou reações adversas dos medicamentos ao tratamento com COVID-19 - hidroxicloroquina sulfato (Reuquinol, 400 $\mathrm{mg} / \mathrm{dia}$ ), Ceftriaxona sódica (2 g/dia), e azitromicina (Zitroma 500 mg/dia).

Além dessas alterações, distúrbios na articulação temporomandibular e bruxismo foram observadas em pacientes com COVID-19, que podem estar associados com o seu estado emocional ${ }^{14}$. Logo, lesões traumáticas associadas ao estresse, ansiedade e hábitos deletérios, devem ser consideradas no diagnóstico diferencial de lesões orais provocadas pelo vírus SARS-CoV-2르. Chauhan et al. $\frac{6}{}$ constatou também, que pacientes com câncer podem ser mais suscetíveis à infecção por SARS-CoV-2 devido a uma maior carga viral nesses pacientes e sua condição imunológica suprimida concernente aos efeitos da terapia antineoplásica.
O tratamento multidisciplinar aos pacientes com COVID-19, que inclua os cirurgiões-dentistas, faz-se necessário. $O$ exame bucal não deve ser negligenciado, especialmente aos pacientes internados, haja vista a necessidade de suporte, controle da dor e qualidade de vida para esses pacientes ${ }^{20}$. A presença dos cirurgiões-dentistas no ambiente hospitalar têm sido essencial neste período de pandemia, pois sua assistência favorece aos cuidados orais, e principalmente no planejamento da adequação do meio bucal para os indivíduos internados, podendo implicar em um menor tempo de internação e redução dos agravos a saúde oral que a entubação e ventilação mecânica podem provocar $\stackrel{23}{ }$.

Nota-se que o significado epidemiológico das manifestações orais permanece incerto, necessitando de estudos observacionais mais amplos para revelar a prevalência e início desses sintomas 2 202027. As evidências disponíveis ainda são escassas e suas interpretações são limitadas por potenciais vieses e situação de confusão. Os estudos não fornecem evidência epidemiológica convincente para uma associação causa-efeito entre a infecção por COVID-19 e o aparecimento de lesões orais, entretanto, a possibilidade ainda não pode ser descartada.

\section{Conclusão}

Diante do contexto da pandemia do COVID-19, ressalta-se a importância de um minucioso exame clínico pelo cirurgião-dentista, pois uma anamnese e um exame físico bem acurado/executado são fundamentais para o diagnóstico e tratamento das possíveis alterações orais. As principais manifestações bucais descritas na literatura foram infecções fúngicas e virais (HSV-1), ulcerações orais inespecíficas, erupções fixas de medicamentos, disgeusia, xerostomia ligada à diminuição do fluxo salivar e gengivite.

\section{Contribuições dos autores}

Santos Júnior JCC participou da concepção, delineamento, busca e análise bibliográfica da pesquisa, interpretação dos resultados e redação do artigo científico. Souza MS, Santos VS, Pires ALPV, Almeida CBS participaram da concepção, interpretação dos resultados, redação do artigo científico. Carvalho JMS participou da redação do artigo científico. 


\section{Conflitos de interesses}

Nenhum conflito financeiro, legal ou político envolvendo terceiros (governo, empresas e fundações privadas, etc.) foi declarado para nenhum aspecto do trabalho submetido (incluindo, mas não se limitando a subvenções e financiamentos, participação em conselho consultivo, desenho de estudo, preparação de manuscrito, análise estatística, etc.).

\section{Referências}

1. World Health Organization (WHO). Folha informativa COVID-19 (doença causada pelo novo coronavírus) [Internet]. 2020. [acesso em 2020 jul 27]. Disponível em: https://www.paho.org/bra/index.php?option=com content\&view $=$ article\&id=6101: covid1 $19 \&$ ltemid $=875$

2. Santos JA, Normando AGC, Silva RLC, Paula RM, Cembranel AC, Santos-Silva AR et al. Oral mucosal lesions in a COVID-19 patient: New signs or secondary manifestations. Int J Infect Dis. 2020;97(9):326-28. http://dx.doi.org/10.1016/j.ijid.2020.06.012

3. Mallineni SK, Innes NP, Raggio DP, Araujo MP, Robertson MD, Jayaraman J. Coronavirus disease (COVID-19): Characteristics in children and considerations for dentists providing their care. Int J Paediatr Dent. 2020;30(3):245-50. http://dx.doi.org/10.1111/ ipd. 12653

4. Chen N, Zhou M, Dong X, Qu J, Gong F, Han Y et al. Epidemiological and clinical characteristics of 99 cases of 2019 novel coronavirus pneumonia in Wuhan, China: a descriptive study. Lancet. 2020;395(10223):507-513. http://dx.doi. org/10.1016/S0140-6736(20)30211-7

5. Chaux-Bodard A-G, Deneuve S, Desoutter A. Oral manifestation of Covid-19 as an inaugural symptom. J Oral Med Oral Surg. 2020;26(2):18. http://dx.doi.org/10.1051/mbcb/2020011

6.Chauhan A, Ghoshal S, Pal A. Increased susceptibility of SARS-CoV2 infection on oral cancer patients; cause and effects: An hypothesis. Med Hypotheses. 2020;144:1-4. http://dx.doi. org/10.1016/j.mehy.2020.109987

7.Biadsee A, Biadsee A, Kassem F, Dagan O, Masarwa S, Ormainer Z. Olfactory and oral manifestations of COVID-19: Sex-related symptoms - A potential pathway to early diagnosis. Otolaryngol Head Neck Surg. 2020;00:1-7. http://dx.doi. org/10.1177/0194599820934380

8. Rodriguez MD, Romera AJ, Villarroel M. Oral manifestations associated to covid-19. Oral Dis. 2020;00:1-3. http://dx.doi. org/10.1111/odi.13555

9. Vieira AR. Oral manifestations in coronavírus disease 2019 (COVID-19). Oral Dis. 2020;26(5):1. http://dx.doi.org/10.1111/ odi.13463
10. Herrera D, Serrano J, Roldán S, Sanz M. Is the oral cavity relevant in SARS-Cov-2 pandemic?. Clin Oral Investig. 2020;24(8):2925-30. http://dx.doi.org/10.1007/s00784-020-03413-2

11. Song J, Li Y, Huan X, Chen Z, Li Y, Liu C et al. Systematic analysis of ACE2 and TMPRSS2 expression in salivary glands reveals underlying transmission mechanism caused by SARS-CoV-2. J Med Virol. 2020;10.1002. http://dx.doi.org/10.1002/jmv.26045

12. Sinadinos A, Shelswell J. Oral ulceration and blistering in patients with COIVD-19. Evid Based Dent. 2020;21(2):49. http:// dx.doi.org/10.1111/odi.13382

13. Nuno-Gonzalez A, Martin-Carrillo P, Maletsky K, Rios MDM, Mañas $\mathrm{CH}$, Almazan JA et al. Prevalence of mucocutaneous manifestations, oral and palmoplantar findings in 666 patients with COVID-19 in a field hospital in Spain. Br J Dermatol. 2020;00:1-6. http://dx.doi.org/10.1111/bjd.19564

14. Xu H, Zhong L, Deng J, Peng J, Dan H, Zeng X et al. High expression of ACE2 receptor of 2019-nCoV on the epithelial cells of oral mucosa. Int J Oral Sci. 2020;12:8. http://dx.doi.org/10.1038/ s41368-020-0074-X

15. Odeh ND, Babkair H, Abu-Hammad S, Borzangy S, AbuHammad A, Abu-Hammad O. COVID-19: Present and future challenges for dental practice. Int J Environ Res Public Health. 2020;17(9):3151. http://dx.doi.org/10.3390/ijerph17093151

16. Riad A, Klugar M, Krsek M. COVID-19 - Related oral manifestations: Earlys Disease Features?.Oral Dis. 2020;00:1-6. http://dx.doi.org/10.1111/odi.13516

17. Tuñon-Chávez $M$, Castro-Ruiz C. Desafíos de la odontologia frente a la pandemia del COVID-19. Int J Odontostomat. 2020;14(3):325-26. http://dx.doi.org/10.4067/S0718381X2020000300325

18. Abanoub R, Marwa G. Comment on: Oral manifestation of COVID-19 as an inaugural symptom?. J Oral Med Oral Surg. 2020;26(2):19. http://dx.doi.org/10.1051/mbcb/2020020

19. Al-Khatib A. Oral manifestations in COVID-19 patients. Oral Dis. 2020;00:1-2. http://dx.doi.org/10.1111/odi.13477

20. Santos JA, Normando AGC, Silva RLC, Azevedo AC, Canto GL, Sugaya, $\mathrm{N}$ et al. Oral Manifestations in Patients with COVID-19: A Living Systematic Review. J Dent Res. 2020:11;1-14. http://dx. doi. org/10.1177/0022034520957289

21. Abu-Hammad S, Dar Odeh N, Abu-Hammad O. SARS-CoV-2 and oral ulcers: A causative agent or a predisposing factor?. Oral Dis. 2020;00:1-2. http://dx.doi.org/10.1111/odi.13498

22. Varadarajan S, Balaji TM, Sarode SC, Sarode GS, Sharma NK Gondivkar $S$ et al. EMMPRIN/BASIGIN as a biological modulator of oral cancer and COVID-19 interaction: Novel propositions. Med Hypotheses. 2020;143:1-5. http://dx.doi.org/10.1016/j. mehy.2020.110089 
23. Oliveira HAG, Batista LM, Vasconcelos AS, Fernandes DBS, Cavalcanti UDNT. Mudanças da atuação multiprofissional e pacientes com COVID-19 em unidades de terapia intensiva. Health Residencies Journal-HRJ. 2020;1(7):32-51.

24. Wang C, Wu H, Ding X, Ji H, Jiao P, Song H et al. Does infection of 2019 novel coronavírus cause acute and/or chronic sialadenitis. Med Hypotheses. 2020;140:1-5. http://dx.doi.org/10.1016/j. mehy.2020.109789

25. Ansari R, Gheitani M, Heidari F, Heidari F. Oral cavity lesions as a manifestation of the novel vírus (COVID-19): A letter-to-editor. Oral Dis. 2020;00:1-6. http://dx.doi.org/10.1111/odi.13465
26. Sakaida T, Isao T, Matsubara A, Nakamura M, Morita A. Unique skin manifestations of COVID-19: Is drug eruption specific to COVID-19?. J Dermatol Sci. 2020;99(1):62-64. http://dx.doi. org/10.1016/j.jdermsci.2020.05.002

27. Patel J, Woolley J. Necrotizing periodontal disease: Oral manifestation of Covid-19. Oral diseases. 2020;00:1-2. http:// dx.doi.org/10.1111/odi.13462 